\title{
A Case Report of Right Atrial Cardiac Myxoma
}

\section{Nourhan Chaaban $\mathrm{MD}^{1 *}$, Akash Joshi $\mathrm{MD}^{2}$, Shilpa Kshatriya MD ${ }^{1,2}$}

${ }^{1}$ Department of Internal Medicine, University of Kansas-Wichita, Wichita, USA

${ }^{2}$ Department of Radiology, University of Kansas-Wichita, Wichita, USA

${ }^{3}$ Heartland Cardiology, Wichita, USA

*Corresponding Author: Nourhan Chaaban MD, Department of Internal Medicine, University of Kansas-Wichita, Wichita, USA

Received date: 30 July 2021; Accepted date: 07 August 2021; Published date: 11 August 2021

Citation: Chaaban N, Joshi A, Kshatriya S (2021) A Case Report of Right Atrial Cardiac Myxoma. J Med Case Rep Case Series 2(9): https://doi.org/10.38207/jmcrcs20210114

Copyright: (C) 2021 Nourhan Chaaban MD. This is an open-access article distributed under the terms of the Creative Commons Attribution License, which permits unrestricted use, distribution, and reproduction in any medium, provided the original author and source are credited.

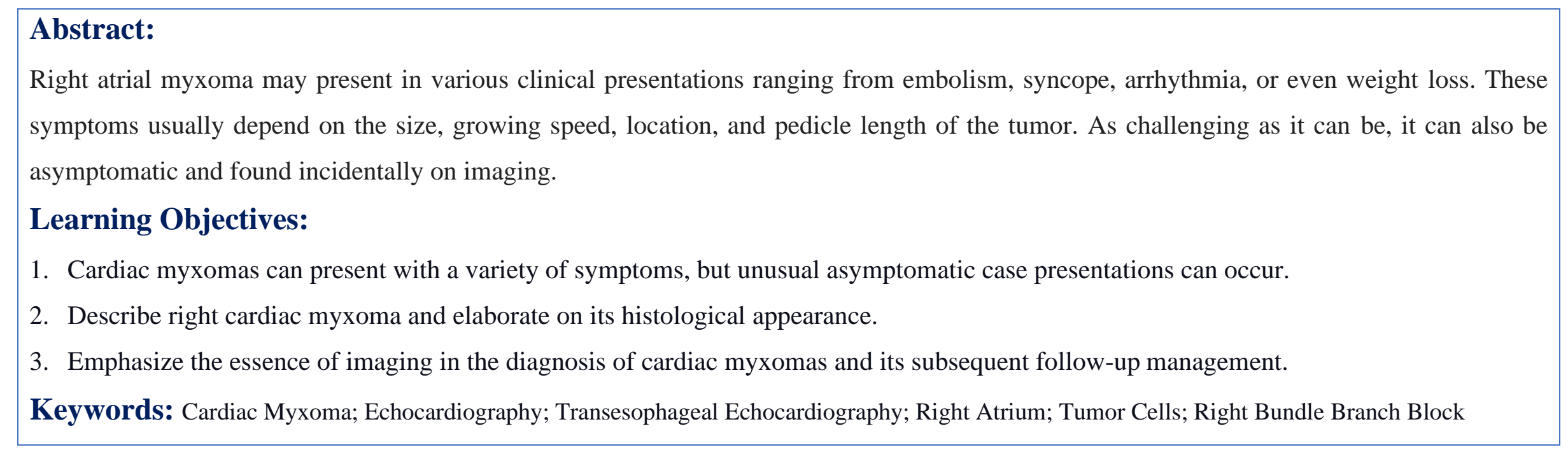

\section{Introduction}

Myxomas are the most common benign primary cardiac tumors. Over $70 \%$ of all cardiac myxomas originate from the left atrium (LA) and $18 \%$ from the right atrium (RA) [1]. Few cases report right atrial myxoma and its clinical presentation. We report here an atrial myxoma case that was found incidentally on imaging.

\section{Case Report}

A 55-year-old female patient with mild intermittent asthma presented to the local emergency room with symptoms of gastroenteritis. She also had symptoms of shortness of breath and coughing on moderate exertion, chest tightness, and occasional dizziness. Her cough would often occur while laying down to sleep and wake her up. She denied exertional chest pain, paroxysmal nocturnal dyspnea, syncope, and

leg swelling. CT abdomen and pelvis with contrast was performed given her abdominal complaints and this showed a filling defect within the right atrium which appeared to enhance on delayed imaging concerning intra-atrial mass, representing a myxoma [Figure 1].

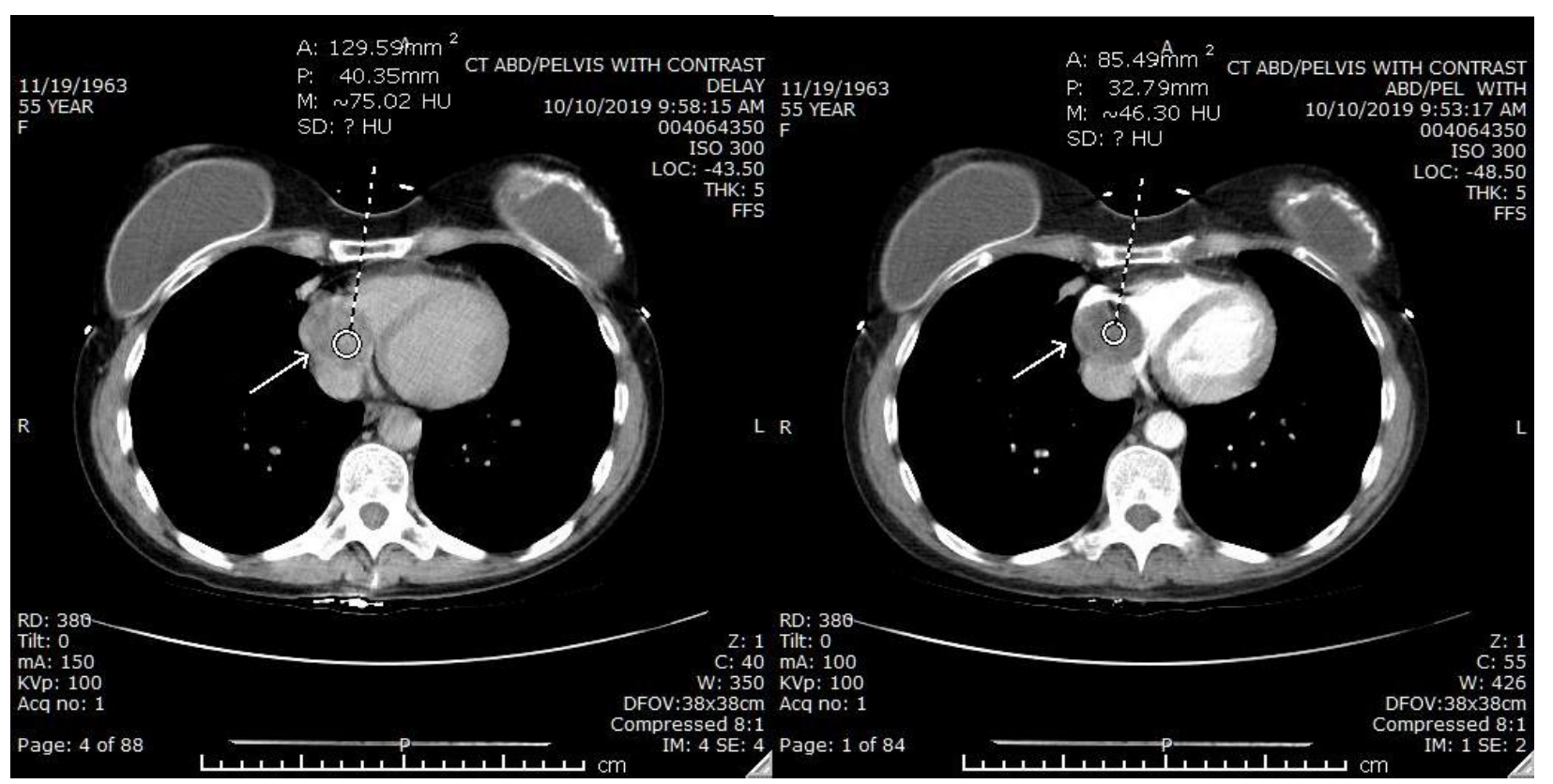

Figure 1: CT abdomen and pelvis with contrast showing a filling defect:

(a) within the right atrium which appeared to enhance on delayed imaging (b) concerning intra-atrial mass, representing a myxoma. 
The patient had no risk factors for coronary heart disease (CHD), such as hypertension, hyperlipidemia, diabetes, smoking, or family history. On initial encounter, her blood pressure was 118/68 mmHg, her heart rate was 98 beats/min, orthostasis vitals were negative. Physical examination was unremarkable. An electrocardiogram showed sinus rhythm with an incomplete right bundle branch block.

An echocardiogram showed a large atrial mass, likely myxoma measuring $4.5 \times 4.4 \mathrm{~cm}$, grade 1 diastolic dysfunction, global left
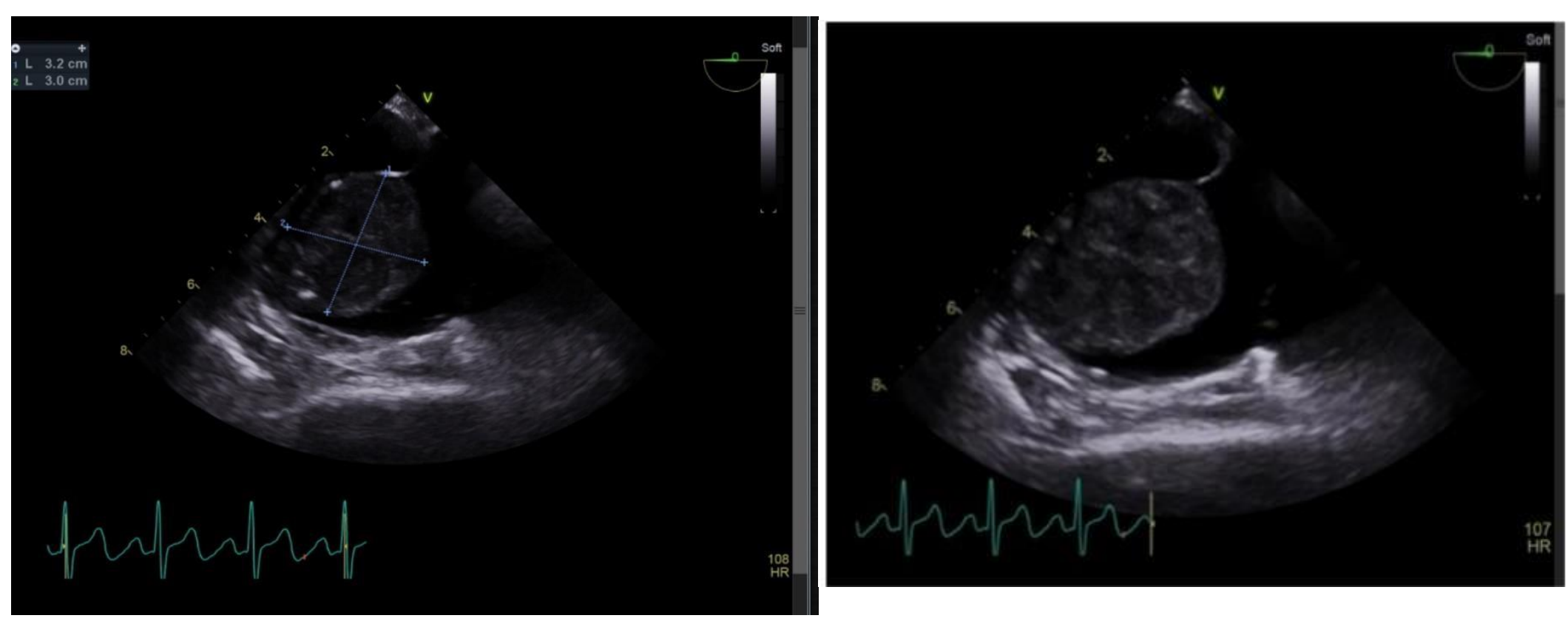

Figure 2: TEE showing normal left ventricular systolic function with an estimated ejection fraction of 55-60\%, mild mitral regurgitation, and large myxoma in the right atrium, measuring $3.0 \mathrm{x} 3.2 \mathrm{~cm}$.
The patient was scheduled for a myxoma removal operation. Under general anesthesia and by being connected to the cardiopulmonary bypass circuit, the myxoma was dissected off the lateral wall of the right atrium using Metzenbaum scissors and Penfield dissector.

Pathology under microscopic examination showed a cardiac myxoma, with epithelioid and spindle tumor cells forming nests, focally circulating vascular spaces. Tumor cells showed mild nuclear polymorphisms, with fine chromatin patterns, inconspicuous nucleolus, and indistinct cell borders with no mitotic activities or tumor necrosis. The background showed abundant eosinophilic myxoid/chondroid substances, and with heavy pigmentation of hemosiderin. Areas of the tumor also showed dense and hyalinized fibrosis with focal ossifications. The surface of the tumor was mostly

\section{Discussion}

Cardiac myxomas are noncancerous primary tumors of the heart and constitute about $50 \%$ of all primary heart tumors [2]. They most commonly occur in women and can present with multiple widespread clinical scenarios ranging from cardiac to extra-cardiac manifestations. There are different clinical symptoms, from asymptomatic to constitutional symptoms such as fever, arterial embolization, endocrine dysfunction, syncope, and even sudden death [3-4].

The most common location of myxomas is in the left atrium, classically at the fossa ovalis border of the interatrial septum [5] or at the mitral annulus [6]. The second most common location forcardiac myxomas is the right atrium with the most common site of attachment ventricular hypokinesia with an ejection fraction of $40 \%$, and mild mitral regurgitation.

Transesophageal echocardiography was performed to evaluate the origin of the cardiac mass [Figure 2]. It showed normal left ventricular systolic function with an estimated ejection fraction of 55$60 \%$, mild mitral regurgitation, and large myxoma in the right atrium, measuring $3.0 \times 3.2 \mathrm{~cm}$. The patient underwent resection of the right atrial mass. eroded, showing patchy areas of intense chronic lymphoplasmacytic inflammations. Further well-controlled special stain (1a) with Mucicarmine and Alcian blue at pH 2.5 highlighted the background of myxoid materials. A stain for iron highlighted the numerous chunky hemosiderin pigments. Further, well-controlled immunohistochemical stains (1A) with CK7, CD34, and CD68, showed the following: CK7 highlighted the tumor spindle cells and focal epithelioid cells, CD43 highlighted the capillary vessel endothelial cells and the partially surviving endocardial endothelial cells, and CD68 stain showed many histiocytes and monocytes from the inflammatory cell populations.

A follow-up echocardiogram was performed, and it showed anormal ejection fraction of $55 \%$ with no residual right atrial mass.

being the interatrial septum, followed by the vena cava, at or nearthe coronary sinus [6].

Myxomas are polypoid, round, or oval and are usually gelatinous, and can have a lobulated surface. Right atrial myxomas tend to be more sessile than left atrial myxomas and can present with acute right heart failure, pulmonary embolism, and even systemic embolization in the presence of a patent foramen ovale or can be asymptomatic. Tricuspid regurgitation can also occur [7]. Our patient had mild cardiac symptoms but her myxoma was discovered incidentally on abdominal CT imaging.

The diagnostic modality of choice is an echocardiography, which identifies the size, site, attachment, mobility, and grossly 
differentiates the myxoma from vegetation or a thrombus [8]. Transesophageal echocardiography is most selected. The sensitivity of transthoracic echocardiography in diagnosing myxoma is $95 \%$, and the sensitivity in transesophageal echocardiography is $100 \%$

\section{Conclusion}

Cardiac myxoma is a benign tumor that requires high clinical suspicion. Most cases are diagnosed incidentally on imaging. Twodimensional echocardiography, including the transesophageal approach, is the technique of choice for diagnosis and follow-up of

Conflict of interest: The authors declare that there is no conflict.

\section{References}

1. Dinesh Kumar US, Wali M, Shetty SP, Sujay KR (2019) Left atrial myxoma - A tumor in transit. Ann Card Anaesth. 22(4): 432-434.

2. Ha JW, Kang WC, Chung N, Chang BC, Rim SJ, et al. (1999) Echocardiographic and morphologic characteristics of left atrial myxoma and their relation to systemic embolism. Am J Cardiol. 83(11): 1579-82, A8.

3. Yuan S-M (2015) Infected cardiac myxoma: An updated review. Braz J Cardiovasc Surg. 30(5): 571-578.

4. Thyagarajan B, Kumar MP, Patel S, Agrawal A (2017) Extracardiac manifestations of atrial myxomas. J Saudi Heart Assoc. 29(1): 37-43.

5. Lazaros G, Masoura C, Brili S, Stavropoulos G, Kafiri G, et al. (2013) Large left atrial myxoma in an oligosymptomatic young woman. Hellenic J Cardiol. 54(1): 60-3.
[9].Atrial myxoma is a benign tumor. The only effective treatment for it is surgical removal. Some have a low recurrence rate. That is why it is crucial to order echocardiography for its early detection.

this tumor [10]. Tumor resection is the gold standard of treatment. Follow-up echocardiography imaging is needed to monitor cardiac function and recurrence rate.

Acknowledgments: This report has not been presented elsewhere. We wish to thank all the co-medical staff involved in the treatment.

6. Li H, Guo H, Xiong H, Xu J, Wang W, et al. (2016) Clinical features and surgical results of right atrial myxoma: Surgical results for right atrial myxoma. J Card Surg. 31(1): 15-7.

7. Oshiumi M, Hashimoto K, Sasaki T, Takakura H, Hachiya T, et al. (2001) Right atrial myxoma complicated with pulmonary embolism. Jpn J Thorac Cardiovasc Surg. 49(7): 449-52.

8. Swartz MF, Lutz CJ, Chandan VS, Landas S, Fink GW (2006) Atrial myxomas: pathologic types, tumor location, and presenting symptoms. J Card Surg. 21(4): 435-40.

9. Al Zahrani IM, Alraqtan A, Rezk A, Almasswary A, Bella A (2014) Atrial myxoma related myocardial infarction: Case report and review of the literature. J Saudi Heart Assoc. 26(3): 166-9.

10. Lone RA, Ahanger AG, Singh S, Mehmood W, Shah S, et al. (2008) Atrial myxoma: trends in management. Int J Health Sci (Qassim). 2(2): 141-51. 